\title{
ON THE KELVIN-HELMHOLTZ INSTABILITY OF THE EARTH'S MAGNETOPAUSE
}

\author{
R. S. B. ONG and N. RODERICK \\ Aerospace Engineering Department, The University of Michigan, \\ Ann Arbor, Michigan 48104, U.S.A.
}

(Recelved in final form 17 August 1971)

\begin{abstract}
The effect of the finite thickness of the shear layer on the Kelvin-Helmholtz instability of the Earth's magnetopause boundary is investigated. The thickness of the layer stabilizes the boundary with respect to short wavelength perturbations, which were previously found to be unstable in the zero thickness analysis. Compressibility effects further stabilize the layer. The effects of the magnetic field on the instability are also discussed.
\end{abstract}

\section{INTRODUCTION}

The hydromagnetic Kelvin-Helmholtz $(\mathrm{K}-\mathrm{H})$ instability has been discussed by various authors in connection with the solar wind and its interaction with the Earth's magnetic field. Fejer (1964) and McKenzie (1970) considered the K-H instability of the magnetopause in regard to the viscous interaction between the solar wind and the magnetosphere. The magnetopause instability phenomenon was also used to explain the semi-annual magnetic field variation (Boller and Stolow, 1970), and the occurrence of ultra low frequency micropulsations in the magnetic field (Dungey and Southwood, 1970). In regard to the physics of the solar wind itself the instability has been discussed in connection with solar wind heating and the smoothing of velocity gradients between fast and slow streams (Coleman, 1969; Jokipii and Davis, 1969).

Southwood (1968) showed that the magnetopause is unstable at low and middle latitudes even for small values of the flow velocity. For these cases the flow velocity is not closely aligned with the Earth's magnetic field and has a large component in the equatorial plane. In these low latitude regions we find a large north-south oriented magnetic field inside the magnetosphere. On the other hand, the field in the magnetosheath is much smaller and oriented in a different direction. The instability was shown to develop in regions along the equatorial plane just away from the subsolar point. The flow velocity in these regions is small compared to the local speed of sound.

Lerche (1966) showed that the hydromagnetic analysis of the $\mathrm{K}-\mathrm{H}$ instability of shear layers of zero thickness leads to an inconsistency. The stability criterion depends on the phase velocity of the various modes and therefore the highest growth rate occurs for the shortest wavelength disturbances. However, for very short wavelength perturbations the thickness of the shear layer should be taken into account. In this paper we study the effect of the finite thickness of the shear layer on the $\mathrm{K}-\mathrm{H}$ instability of the magnetopause. In the following section we introduce the model and formulate the problem. Since the flow velocity in the region of instability is small compared to the sound speed we first perform the incompressible flow analysis. The effect of compressibility is then shown next.

In order to analyze the magnetosphere boundary instability problem properly Lerche (1966) also felt that the collisionless Vlasov equation together with the Maxwell's equations should be used. As we have no way of determining the form of the particle distribution function in the turbulent magnetosheath region this approach is theoretically very difficult, if not impossible. Thus we shall use a hydromagnetic model and limit ourselves to an order of magnitude estimate of the mascroscopic behavior of the magnetosphere boundary. 


\section{FORMULATION OF THE PROBLEM}

Consider a shear layer of thickness $2 d$ in the $y-z$ plane of a Cartesian coordinate system as shown in Fig. 1.

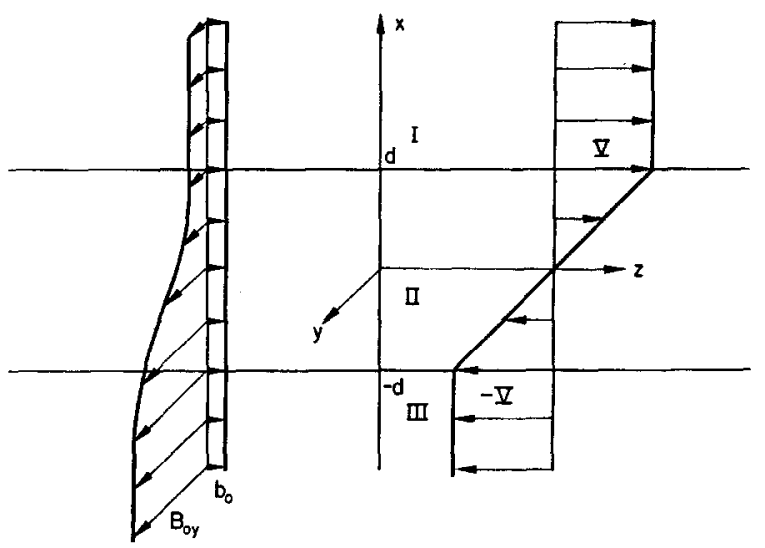

Fig. 1. Shear LAyer MOdel.

The layer separates two regions of infinitely conducting fluids. In these regions the fluid and electromagnetic field properties are constant; they vary only inside the layer as shown in Fig. 1. The magnetic field is tangential to the layer and its variation is arbitrary. The fluid velocity has been transformed in a way such that the constant flow velocities in regions I and III may be given by $v_{0 z}= \pm V$ respectively. Its variation through the layer is assumed to be linear. The equilibrium magnetic field is taken to be of the form

$$
\mathbf{B}_{0}=B_{0 y}(x) \hat{e}_{y}+b_{0} \hat{e}_{z}
$$

where $b_{0}$ is constant. As our model we shall use the ideal hydromagnetic equations with an isotropic pressure tensor. The fundamental equations are:

$$
\begin{aligned}
\frac{\partial \rho}{\partial t}+\operatorname{div} \rho \mathbf{v} & =0 \\
\rho\left(\frac{\partial \mathbf{v}}{\partial t}+\mathbf{v} \cdot \operatorname{grad} \mathbf{v}\right) & =-\operatorname{grad} p+\mathbf{j} \times \mathbf{B} \\
\mathbf{E}+\mathbf{v} \times \mathbf{B} & =0 \\
\operatorname{curl} \mathbf{E} & =-\frac{\partial \mathbf{B}}{\partial t} \\
\operatorname{curl} \mathbf{B} & =\mu_{0} \mathbf{j} \\
\operatorname{div} \mathbf{B} & =0 .
\end{aligned}
$$

The electric field may be eliminated in equations (3) and (4) to obtain the magnetic field transport equation

$$
\frac{\partial \mathbf{B}}{\partial t}+\mathbf{v} \cdot \operatorname{grad} \mathbf{B}=\mathbf{B} \cdot \operatorname{grad} \mathbf{v}+\mathbf{B} \operatorname{div} \mathbf{v} .
$$

The above set of equations may then be closed by the specification of an appropriate pressure density relation. 
We now impose a small amplitude disturbance such that the perturbation quantities vary as $\exp i\left(k_{y} y+k_{z} z-\omega t\right)$. The fundamental equations above may then be linearized and they take the form:

$$
\begin{gathered}
-i \omega^{*} \rho_{1}+\rho_{0}\left(\operatorname{div} v_{1}\right)+v_{1 x} \frac{\mathrm{d} \rho_{0}}{\mathrm{~d} x}=0 \\
-i \omega^{*} v_{1 x}=-\frac{1}{\rho_{0}} \frac{\mathrm{d} p_{1}}{\mathrm{~d} x}-\frac{1}{\rho_{0} \mu_{0}}\left\{\frac{\mathrm{d}}{\mathrm{d} x}\left(B_{0} b_{y}\right)+b_{0}\left(\frac{\mathrm{d} b_{z}}{\mathrm{~d} x}-i k_{z} b_{x}\right)-i k_{y} B_{0 y} b_{x}\right\} \\
-i \omega^{*} v_{1 y}=-\frac{i}{\rho_{0}} k_{y} p_{1}+\frac{b_{x}}{\rho_{0} \mu_{0}} \frac{\mathrm{d} B_{0 y}}{\mathrm{~d} x}-\frac{i b_{0}}{\rho_{0} \mu_{0}}\left(k_{y} b_{z}-k_{z} b_{y}\right) \\
-i \omega^{*} v_{1 z}+v_{1 x} \frac{\mathrm{d} v_{0 z}}{\mathrm{~d} x}=-\frac{i}{\rho_{0}} k_{z} p_{1}+\frac{i B_{0 y}}{\rho_{0} \mu_{0}}\left(k_{y} b_{z}-k_{z} b_{y}\right) \\
-i \omega^{*} b_{x}=i b_{0} k_{z} v_{1 x} \\
-i \omega^{*} b_{y}+v_{1 x} \frac{\mathrm{d} B_{0 y}}{\mathrm{~d} x}=i b_{0} k_{z} v_{1 y}+B_{0 y} \operatorname{div} \mathbf{v}_{1} \\
\frac{\mathrm{d} b_{x}}{\mathrm{~d} x}+i k_{y} b_{y}+i k_{z} b_{z}=0
\end{gathered}
$$

where $\omega^{*} \equiv \omega-k_{z} v_{0 z}(x)$.

In the equilibrium state the pressure balance across the layers is given by

$$
\left(p_{0}+\frac{B_{0}^{2}}{2 \mu_{0}}\right)_{\mathrm{I}}=\left(p_{0}+\frac{B_{0}{ }^{2}}{2 \mu_{0}}\right)_{\mathrm{II}}=\left(p_{0}+\frac{B_{0}{ }^{2}}{2 \mu_{0}}\right)_{\mathrm{III}} .
$$

As mentioned in the Introduction, instability of the magnetopause may occur in regions where the flow velocity in the magnetosheath is small. This can take place not too far away from the subsolar point. Thus at the point where the instability occurs the flow speed will be very small compared to, say, the local acoustic speed, i.e. $|V| \ll c_{s}$, where $c_{\text {g }}$ is the local speed of sound. Consequently we shall first consider the incompressible case and later discuss the effects of compressibility.

\section{INCOMPRESSIBLE FLOW LIMIT}

For incompressible flow $\rho_{0}=$ constant, and the linearized continuity equation reduces to

$$
\frac{\mathrm{d} v_{1 x}}{\mathrm{~d} x}+i k_{y} v_{1 y}+i k_{z} v_{1 x}=0 .
$$

In this case the system of equations (9)-(15) constitute a complete set. The configuration shown in Fig. 1 approximates the flow and magnetic field conditions on the dusk side in the equatorial plane. The magnetosphere with its relatively large magnetic field is represented by region III. Thus the magnetic field increases from a small value $B_{01}$ in the magnetosheath (region I) to the larger value $B_{03}$ in the magnetosphere, with the arbitrary variation taking place inside the magnetopause region which is represented by the finite thickness layer. The equilibrium magnetic field also has a small component tangential to the flow velocity vector. This is represented by the constant $z$-component $b_{0}$ shown in Fig. 1. Since the flow is nearly normal to the magnetic field in the magnetosphere, we assume 
$b_{0} \ll B_{03}$. We shall consider the case of the most unstable mode; this propagates in a direction perpendicular to the magnetic field in the magnetosphere (Southwood, 1968). Thus we let

$$
\mathbf{k} \simeq k_{z} \hat{e}_{z} .
$$

From the set of equations above we obtain after some algebraic operations the following equation:

$$
\begin{aligned}
\omega^{* 2}\left(\omega^{* 2}-k_{z}{ }^{2} c_{a}{ }^{2}\right) & \frac{\mathrm{d}^{2} v_{1 x}}{\mathrm{~d} x^{2}}-2 k_{z}^{3} c_{a}{ }^{2} \frac{\mathrm{d} v_{0 z}}{\mathrm{~d} x} \omega^{*} \frac{\mathrm{d} v_{1 x}}{\mathrm{~d} x} \\
- & {\left[k_{z} \omega^{*}\left(k_{z} \omega^{*}-\frac{\mathrm{d}^{2} v_{0 z}}{\mathrm{~d} x^{2}}\right)\left(\omega^{* 2}-k_{z}{ }^{2} c_{A}{ }^{2}\right)+2 k_{z}{ }^{4} c_{a}{ }^{2}\left(\frac{\mathrm{d} v_{0 z}}{\mathrm{~d} x}\right)^{2}\right] v_{1 x}=0 }
\end{aligned}
$$

where $c_{a}{ }^{2}=b_{0}{ }^{2} / \rho_{0} \mu_{0}$. This equation is valid everywhere and the boundary conditions are

$$
v_{1 x} \rightarrow 0 \text { as } x \rightarrow \pm \infty .
$$

At the interface the perpendicular displacement of the layer, the normal component of the magnetic field, and the normal stress must be continuous. Assuming small amplitude displacement of the interface we can approximately carry out the matching at $x= \pm d$.

In the case of a zero thickness interface a similar analysis yields a dispersion relation of the form

$$
\omega^{2}=k^{2}\left(c_{a}^{2}-V^{2}\right) \text {. }
$$

Thus in this case the flow is unstable when $V>c_{a}$, and assuming

$$
\omega=\omega_{R}+i \gamma
$$

the growth rate of the instability is given by

$$
\gamma=k V\left(1-\frac{c_{a}}{V}\right)^{1 / 2} ; V>c_{a} .
$$

Note that the largest growth rate occurs for disturbances with $k \rightarrow \infty$ (Lerche, 1966).

Let us now return to the case where the interface thickness is finite. Outside the layer (regions I and III) the equilibrium flow velocity $v_{0 z}$ is independent of the $x$-coordinate. In these regions the solutions to the differential equations which satisfy the boundary conditions at $x= \pm \infty$ are

$$
\begin{aligned}
v_{1 x} & =A_{1} \exp \left(-k_{z} x\right) \quad \text { for } \quad x>d \\
& =c_{3} \exp \left(k_{z} x\right) \quad \text { for } \quad x \ll d
\end{aligned}
$$

where $A_{1}$ and $C_{3}$ are constants.

Within the interface (region II) the flow velocity $v_{0 x}$ varies with the $x$-coordinate. In this region we define a new variable $\delta$ by

$$
\delta \equiv v_{1 x} / \omega^{*} .
$$

In terms of $\delta$ the differential equation (16) may be written as

$$
\frac{\mathrm{d}}{\mathrm{d} x}\left[\left(\omega^{*^{2}}-k_{z}{ }^{2} c_{a}{ }^{2}\right) \frac{\mathrm{d} \delta}{\mathrm{d} x}\right]-k_{z}{ }^{2}\left(\omega^{*^{2}}-k_{z}{ }^{2} c_{a}{ }^{2}\right) \delta=0
$$


with the boundary conditions

$$
\delta \rightarrow 0 \quad \text { as } \quad x \rightarrow \pm \infty .
$$

We now introduce the transformation

$$
\xi=k_{z} x-\Omega
$$

where $\Omega=\omega \mathrm{d} / V=\Omega_{R}+i \Gamma$. The differential equation (22) then becomes

$$
\frac{d}{d \xi}\left[\left(\xi^{2}-\alpha^{2}\right) \frac{d \delta}{d \xi}\right]-\left(\xi^{2}-\alpha^{2}\right) \delta=0
$$

where

$$
\begin{aligned}
\alpha^{2} & =k^{2} / A^{2} \\
k & =k_{z} d \\
A^{2} & =V^{2} / c_{a}^{2} .
\end{aligned}
$$

The edges of the interface are given by

$$
\begin{gathered}
x=d \rightarrow \xi=\xi_{1}=k_{z} d-\Omega \\
x=-d \rightarrow \xi=\xi_{2}=-k_{z} d-\Omega .
\end{gathered}
$$

Equation (24) is a spheroidal wave equation and its general solution may be obtained by a series expansion of appropriate functions (Morse and Feshbach, 1953). However, since $\xi$ contains $\omega$ the series solution is awkward for the discussion of dispersion relations. Hence it is more convenient to find an approximate solution by means of a WKB procedure. In order to do this we first define the variable $\eta$ by

$$
\mathrm{d} \eta=\mathrm{d} \xi / \xi^{2}-\alpha^{2} .
$$

The differential Equation (24) can then be transformed into standard form:

$$
\frac{\mathrm{d}^{2} \delta}{\mathrm{d} \eta^{2}}-Q^{2}(\eta) \delta=0
$$

where $Q^{2}(\eta)=\xi^{2}(\eta)-\alpha^{2}$. For $Q$ not near zero the asymptotic solution expressed in the $\xi$ variable, is given by

$$
\delta=A_{+}\left(\xi^{2}-\alpha^{2}\right)^{-1 / 2} \exp (\xi)+A_{-}\left(\xi^{2}-\alpha^{2}\right)^{-1 / 2} \exp (-\xi) .
$$

The WKB validity condition implies that the solution given by (26) is a valid one provided

and

$$
\begin{array}{clll}
A^{2} \gg 1 & \text { for } & & k<0(1) \\
k \gg 1 & \text { for } & & A \leqslant 0(1) .
\end{array}
$$

It is interesting to note that (26) becomes an exact solution to the differential equation (24) for $A \rightarrow \infty$. This is the 'vanishing parallel field limit'. It corresponds to $b_{0}=0$; this implies that the magnetic field in the magnetosheath is aligned with the field in the magnetosphere. In this case the results are the same as those obtained by Rayleigh for the hydrodynamic problem.

The condition $A^{2} \gg 1$ above indicates that the flow velocity, while small compared to the acoustic velocity, must be large compared to the Alfvén velocity based upon the parallel magnetic field component $b_{0}$.

An interesting feature of the incompressible flow case is that the relevant differential 
equation and its solution are independent of the variation of $B_{0}$. The magnetic field component perpendicular to the direction of the wave normal of the disturbance and the flow velocity does not affect the stability of the shear layer. The same result was obtained in the case of a zero thickness shear layer with a constant perpendicular magnetic field (Chandrasekhar, 1961).

The dispersion relation is obtained from the condition that the normal displacement, normal component of the magnetic field, and normal component of the stress be continuous at the edges of the shear layer. Continuity of the normal displacement of the interface is the same as requiring $\delta$ to be continuous. The normal stress condition is obtained by integrating Equation (24) across the interface from $\xi_{1,2}-\epsilon$ to $\xi_{1,2}+\epsilon$ and taking the limit as $\epsilon \rightarrow 0$. $\left(\xi_{1,2}\right.$ means the interface edge $\xi_{1}$ or $\left.\xi_{2}\right)$. Hence the interface conditions are

$$
\begin{aligned}
\Delta_{1,2}[\delta] & =0 \\
\Delta_{1,2}\left[\left(\xi^{2}-\alpha^{2}\right) \frac{\mathrm{d} \delta}{\mathrm{d} \xi}\right] & =0
\end{aligned}
$$

where $\Delta_{1,2}[$ ] indicates the jump in the quantity inside the square bracket at the interface edge $\xi_{1}$ and $\xi_{2}$. Since $\xi$ is continuous at $\xi_{1}$ and $\xi_{2}$ the second relation becomes

$$
\Delta_{1,2}\left[\frac{\mathrm{d} \delta}{\mathrm{d} \xi}\right]=0
$$

For $A_{1}, A_{3}, A_{+}$and $A_{-}$non-zero we obtain the following dispersion relation:

$$
\begin{aligned}
& \Omega^{4}+\Omega^{2}\left[k-2 k^{2}\left\{1+\left(1 / A^{2}\right)\right\}-\frac{1}{4}\{1-\exp (-4 k)\}\right] \\
& +k^{4}\left(A^{2}-1\right)^{2} / A^{4}-k^{3}\left(A^{2}-1\right) / A^{2}+\frac{1}{4} k^{2}\{1-\exp (-4 k)\}=0 .
\end{aligned}
$$

For $A \rightarrow \infty$ this reduces to the dispersion relation in the hydrodynamic case with no magnetic field. We evaluated (30) numerically and the typical growth rate versus the dimensionless wave number is shown in Fig. 2. The $c_{a} \neq 0$ growth rate curves shown are for a relatively large value of the Alfvén Mach number based on the parallel component of the magnetic

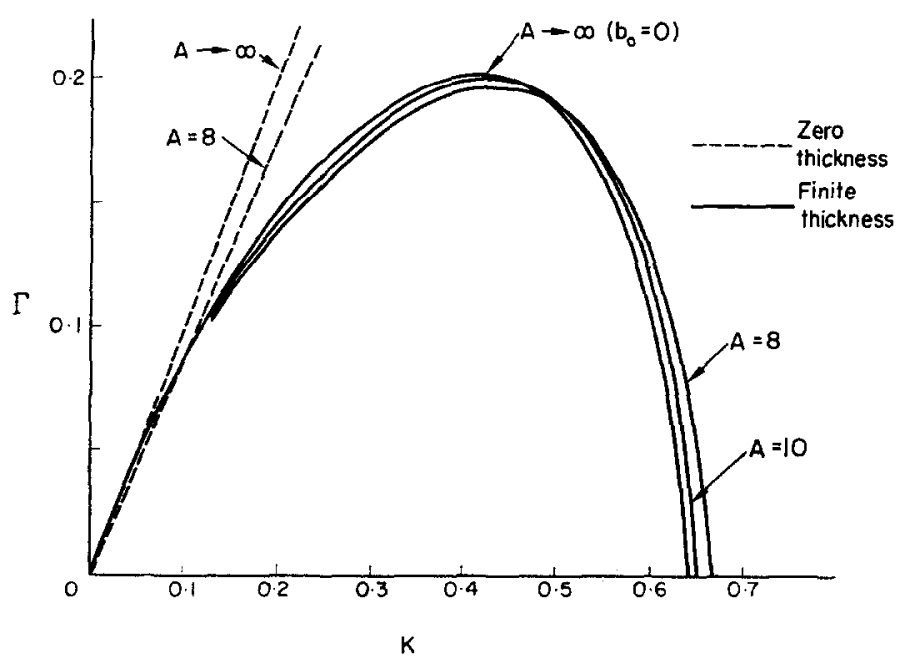

Fig. 2. Growth RATE vs. WAVE NUMBER. EFFECtS OF FINITE LAYER THICKNESS AND PARALlei MAGNETIC FIELD. 
field. The well-known results for the case of a zero thickness shear layer are also shown for comparison. These results show the existence of a critical wave number $k_{0}$ such that for $k>k_{c}$ the growth rate of the disturbance is zero. When we have a nonzero parallel magnetic field $b_{0}$ the growth rate of the instability is reduced. Thus for a shear layer of a certain thickness the parallel component of the magnetic field in this sense tends to stabilize the flow with respect to long wavelength disturbances. However, the presence of $b_{0}$ increases the value of $k_{\mathrm{c}}$ as shown in Fig. 2. Nevertheless, from the WKB approximation, which is valid for arbitrary large values of $k$, the results show that the growth rate of the short wavelength disturbances is zero for all values of the parallel Alfvén Mach number.

In summary, the finite thickness of the shear layer stabilizes it in regard to short wavelength perturbations. With respect to long wavelength disturbances it limits the growth rate to finite values. A parallel magnetic field component $b_{0}$ tends to stabilize the layer even further, but it extends the value of the critical wave number somewhat.

\section{EFFECTS OF COMPRESSIBILITY}

In order to study the effects of compressibility we first introduce the following simplifications to our model. The $y$-component of the magnetic field $B_{0}$ will be taken to be constant, while the $z$-component $b_{0}$ is assumed to be equal to zero. These simplifications do not influence the basic effects of the compressibility of the plasma. Since the density is variable, the full form of the continuity equation as given in (8) must be used. To complete the set of equations we now use the adiabatic equation of state:

$$
\mathrm{d} p=c_{s}^{2} \mathrm{~d} \rho
$$

where $c_{8}$ is the local speed of sound. Following the same procedure as before we now obtain a single equation for the $z$-component of velocity:

$$
\frac{\mathrm{d}^{2} v_{z}}{\mathrm{~d} x^{2}}-\left(k^{2}-\frac{\omega^{* 2}}{c_{M}^{2}}\right) v_{z}=0
$$

where $c_{M}=\left(c_{s}^{2}+c_{A}^{2}\right)^{1 / 2}=$ the magneto-acoustic speed, and $c_{A}^{2}=B_{0}^{2} / \mu_{0} \rho_{0}$. The boundary conditions are

$$
v_{z} \rightarrow 0 \quad \text { as } \quad x \rightarrow \pm \infty
$$

Again we introduce the transformation

and Equation (31) becomes

$$
\xi=k_{z} x-\Omega
$$

$$
\frac{\mathrm{d}^{2} v_{z}}{\mathrm{~d} \xi^{2}}-\left(1-\mu^{2} \xi^{2}\right) v_{s}=0
$$

where $\mu^{2}=M^{2} / k^{2}$ and $M$ is the 'magneto-acoustic' Mach number defined by

$$
M=V / c_{M} .
$$

In regions I and III the velocity is independent of $x$ and the differential equation for $v_{z} \operatorname{can}$ be written as

where

$$
\frac{\mathrm{d}^{2} v_{z}}{\mathrm{~d} \xi^{2}}-\left(1-\mu^{2} \xi_{1,3}^{2}\right) v_{k}=0
$$

$$
\begin{aligned}
& \xi_{1}=k-\Omega \\
& \xi_{3}=-k-\Omega .
\end{aligned}
$$


The solution to Equation (33) which satisfies the appropriate boundary conditions are

$$
\begin{aligned}
v_{z} & =A_{1} \exp \left[-\left(1-\mu^{2} \xi_{1}^{2}\right)^{1 / 2} \xi\right] ; \xi>\xi_{1} \\
& =C_{3} \exp \left[\left(1-\mu^{2} \xi_{3}^{2}\right)^{1 / 2} \xi\right] ; \xi<\xi_{3} .
\end{aligned}
$$

In region II the flow velocity is again a function of $x$ and we must solve Equation (32). A similar equation was obtained by Schuurman (1969) for the case of a compressible flow with no magnetic field. The general solution of (32) may be obtained in term of a series expansion in parabolic cylindrical functions. Similar to the spheroidal wave equation for the incompressible case the functional series solution is inappropriate for the purpose of discussing the dispersion relation. For this reason we shall obtain an approximate solution.

In regard to our magnetopause problem we are particularly interested in the region of low velocity just away from the subsolar point. Hence we shall introduce a 'small magnetoacoustic Mach number' approximation, i.e.

$$
M^{2} \equiv \frac{V^{2}}{C_{M}^{2}} \ll 1
$$

This implies $\mu^{2} \ll 1$. Physically this represents a low Mach number approximation for wave numbers $k \leqslant O(1)$, but it may also represent for $k \gg 1$ a situation where the Mach number is finite.

We now use a singular perturbation method and expand $v_{z}$ in terms of the small parameter $\mu^{2}$ :

$$
v_{z}=v_{z}^{(0)}+\mu^{2} v_{z}^{(1)}+\mu^{4} v_{z}^{(2)}+\ldots
$$

Substituting this in (32) we obtain to zeroth order in $\mu^{2}$ :

first order in $\mu^{2}$ :

$$
\frac{\mathrm{d}^{2} v_{z}^{(0)}}{\mathrm{d} \xi^{2}}-v_{z}^{(0)}=0
$$

$$
\frac{\mathrm{d}^{2} v_{z}^{(1)}}{\mathrm{d} \xi^{2}}-v_{z}^{(1)}=A_{2} \exp (\xi)-C_{2} \exp (-\xi)
$$

etc.

The solution for $v_{z}$ to first order in $\mu^{2}$ is

$$
\begin{aligned}
v_{8}=A_{2} \exp (\xi)\left[1+\mu^{2}\left(-4 \xi^{3}+\right.\right. & \left.\left.6 \xi^{2}-6 \xi+3\right) / 24\right] \\
& +C_{2} \exp (-\xi)\left[1+\mu^{2}\left(4 \xi^{3}+6 \xi^{2}+6 \xi+3\right) / 24\right] .
\end{aligned}
$$

The matching conditions at the interface are again the continuity of the normal displacement, the normal component of the magnetic field, and the normal stress. With the aid of the continuity equation (8) these may be expressed in terms of $v_{z}$ as

$$
\begin{aligned}
& \Delta_{1,2}\left[v_{z}\right]=-i \frac{k v_{x 1,2}}{V \xi_{1,2}} \Delta_{1,2}\left[\frac{\mathrm{d} v_{0 x}}{\mathrm{~d} \xi}\right] \\
& \Delta_{1,2}\left[\frac{\mathrm{d} v_{z}}{\mathrm{~d} \xi}\right]-0 .
\end{aligned}
$$

For $\mu^{2} \ll 1$ these lead to the dispersion relation:

$$
\begin{aligned}
8 k \mu^{2} \Omega^{4}+\Omega^{2}[-4 & +\mu^{2}\left(-1-4 k+8 k^{2}-\frac{10}{3} k^{3}\right] \\
& +(1-2 k)^{2}-\exp (-4 k)-\mu^{2}\left(k^{2}-\frac{4}{3} k^{3}-\frac{8}{3} k^{4}+\frac{8}{5} k^{5}\right)=0 .
\end{aligned}
$$


For $\mu^{2}=0$ this relation reduces to that obtained in the hydrodynamic incompressible case. The dispersion relation (37) is solved numerically and the growth rate of the instability is shown in Fig. 3 as a function of the dimensionless wave number $k$ for a fixed value of $M^{2}$.

The result shows that the finite thickness of the layer does stabilize the short wavelength disturbances which were found to be unstable in the zero-thickness limit. Similar results were obtained by Schuurman (1969) who studied the finite thickness layer with no magnetic

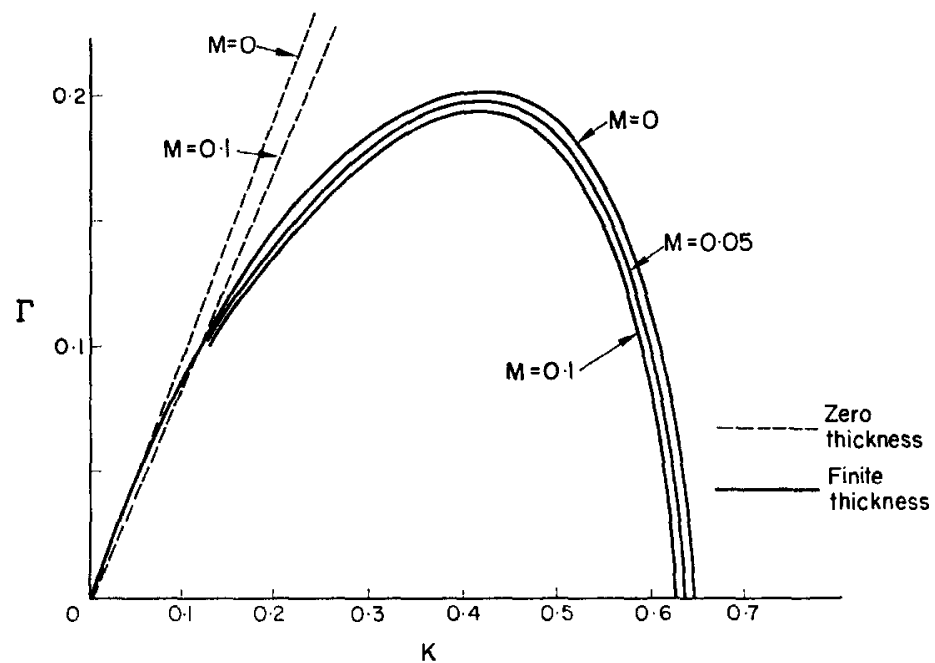

Fig. 3. Growth Rate vs. WAve nUmber. EFFects of finite laypr thickness and COMPRESSIBILITY.

field. Again the growth has a finite value for each wavenumber. Also compressibility effects tend to lower the growth rate of the instability from that for the incompressible case, and it also reduces the value of the critical wave number $k_{c}$. However the perpendicular magnetic field does have an effect when the flow is compressible; it increases the growth rate towards the incompressible value. This is so since $B_{0} \neq 0$ implies $c_{M}>c_{8}$ and $M^{2}$ is then reduced. Hence in this sense the magnetic field tends to be destabilizing. These results agree qualitatively with those obtained when the layer thickness is neglected. For a zero thickness shear layer Miles (1958) found that compressibility effects stabilize the flow, and Fejer (1964) showed that a perpendicular magnetic field tends to be destabilizing. However, the zero thickness assumption always yields an increasing growth rate as the wave number increases. For comparison these are also shown in Fig. 3. When $M$ is not small, then we must have $k \gg 1$ in order for the expansion to be valid. In this case the growth rate is reduced to zero by the finite thickness of the layer.

\section{CONCLUSION}

We have studied two cases of the $\mathrm{K}-\mathrm{H}$ instability which approximate the low latitude magnetopause problem. In both cases the finite thickness of the shear layer is shown to stabilize the short wavelength perturbations, which were previously found unstable in the zero thickness analysis of the hydromagnetic $\mathrm{K}-\mathrm{H}$ problem. Furthermore in the case of incompressible flow the component of the magnetic field perpendicular to the flow velocity and the direction of propagation of the disturbances has no effect on the instability. On the other hand the parallel component of the magnetic field reduces the growth rate of the 
instability. When the variation in the density is taken into account the compressibility effects reduce the growth rate of the instability, but in this case the presence of a perpendicular magnetic field tends to increase the growth rate to its incompressible value.

Acknowledgements-This work was supported by the Air Force Office of Scientific Research under Grant AF-AFOSR-825-67.

\section{REFERENCES}

BolleR, B. R. and Stolov, H. L. (1970). J. geophys. Res. 75, 6073.

Chandrasekhar, S. (1961). Hydrodynamic and Hydromagnetic Stability. Oxford Univ. Press, Oxford.

Coleman, Jr., P. J. (1968). Proc. Symp. on Turbulence of Fluids and Plasmas, p. 333. Polytechnic Press, Brooklyn, N.Y.

Dungey, J. W. and Southwood, D. J. (1970). Space Sci. Rev. 10, 672.

FeJER, J. A. (1964). Physics Fluids 7, 499.

JoxIPI, J. R. and DAVIS, JR. L. (1969). Astrophys. J. 156, 1101.

LERCHE, I. (1966). J. geophys. Res. 71, 2365.

McKenzie, J. F. (1970). Planet. Space Sci. 18, 1.

MILES, J. W. (1958). J. fluid Mech. 4, 538.

Morse, P. M. and Feshbach, H. (1953). Methods of Theoretical Physics Part 1. McGraw-Hill, N.Y.

SchuURman, W. (1969). Rijnhuizen Rep. 69-55, Jutphaas, Netherlands.

SouTHWOOD, D. J. (1968). Planet Space Sci. 16, 587. 